\title{
The Leaky Storage Model for Optimal Multi-service Allocation of Thermostatic Loads
}

\author{
Vincenzo Trovato ${ }^{(1)}$, Simon H. Tindemans ${ }^{(2)}$ and Goran Strbac ${ }^{(3)}$ \\ Department of Electrical and Electronic Engineering - Imperial College London \\ South Kensington Campus, London SW7 2AZ, UK, United Kingdom \\ ${ }^{(1)}$ v.trovato10@imperial.ac.uk, ${ }^{(2)}$ s.tindemans@imperial.ac.uk (corresponding author), ${ }^{(3)}$ g.strbac@imperial.ac.uk
}

\begin{abstract}
Collectively, thermostatically controlled loads (TCLs) offer significant potential for short-term demand response. This intrinsic flexibility can be used to provide various ancillary services or to carry out energy arbitrage. This paper introduces an aggregate description of the flexibility of a heterogeneous TCLs as a leaky storage unit, with associated constraints that are derived from the TCL device parameters and quality of service requirements. In association with a suitable TCL control strategy this enables a straightforward embedding of TCL dynamics in optimisation frameworks. The tools developed are applied to the problem of determining an optimal multi-service portfolio for TCLs. A linear optimisation model is constructed for the optimal simultaneous allocation of frequency services and energy arbitrage. In a case study, optimal service allocations are computed for eight representative classes of cold appliances and the results are validated using simulations of individual refrigerators. Finally, it is demonstrated that clustering of appliances with similar capabilities can significantly enhance the flexibility available to the system.
\end{abstract}

Index Terms-Energy storage, Energy arbitrage, Frequency regulation, Demand side response, Optimization.

\section{INTRODUCTION}

Thermostatically Controlled Loads (TCLs) encompass devices such as refrigerators and space heaters and represent a significant share of the domestic electricity demand. These devices are usually controlled by means of a hysteresis controller with a temperature deadband; relatively small alterations to the regular operating cycle can be tolerated. Hence, TCLs collectively represent a sizable distributed energy resource. If a large population of TCLs were able to adjust its aggregate power consumption on demand, this could offer significant potential benefits [1].

The intrinsic flexibility of TCLs may be used to provide a variety of services to the power system. Time-dependent tariffs may encourage TCLs to realise energy arbitrage, consuming more energy when the electricity prices are low and less when they are high [2]. Other applications include frequency services, where the TCLs provide short-term balancing services, either quasicontinuously to balance a generation and demand mismatch [3] or in response to large frequency deviations [1, 4]. This fastresponding flexibility relieves some conventional generators from being operated part-loaded, improving the efficiency of the system and reducing $\mathrm{CO}_{2}$ emissions [5].

Similar studies, based on energy storage systems, revealed the benefits of optimizing the simultaneous allocation of multiple ancillary services (e.g. [6]). Models that describe the high-level flexibility of storage units are compact and thus benefit from an 
easy embedding in optimization tools. This illustrates the desirability of developing a similar high-level description of aggregate TCL flexibility, for example using an approximate battery model with energy and power constraints [2].

However, constructing a practical high-level storage model for controllable TCLs is not straightforward [7]; it should address the following three challenges. First, the model should have a representation that allows for a direct embedding in optimisation models. Second, aggregate solutions from this model should be achievable by the device controllers. This requirement implies a tight connection between the constraints of the model and the TCL control strategy and its assumed control infrastructure. Many controllers thus impose complex constraints on admissible power profiles, inhibiting the implementation of an accurate high-level representation of TCL flexibility [2]. The third challenge requires the safeguard of the primary function of TCLs: approximately maintaining their respective temperatures around the set-point value. Solutions that cause TCLs to spend all their time at the extremes of their temperature range should therefore be disallowed. Such constraints are unique to TCLs and do not normally apply to storage units.

\section{A. Contributions of this paper}

This paper introduces a high-level description of the aggregate flexibility of TCLs as a dissipative storage unit: the leaky storage model, which simultaneously addresses the challenges described above. The model builds upon an advanced decentralised control strategy we have recently developed [8], which enables accurate decentralised control of aggregate TCL power consumption without violating device constraints. For the purpose of this paper, the controller has been enhanced to use an extended temperature range and for an improved handling of discontinuities (details in the Appendix). Based on the capabilities of the control design, an aggregate TCL model in natural units is formulated in Sec. II.

In Sec. III we demonstrate how the different capabilities of heterogeneous TCLs can be aggregated into a single envelope model that establishes a lowest-common-denominator representation of their flexibility. The decentralised control strategy lets all TCLs respond to a single control signal, which means such a strategy will necessarily not make full use of the capabilities of the most flexible devices. To offset this implicit conservatism, we further present a method to partition a set of heterogeneous devices into clusters with similar flexibility, which can be controlled independently. Finally, it is shown that the aggregate flexibility of a cluster of devices has an intuitive physical interpretation as a 'leaky storage unit'. Besides constraints on power consumption and energy levels, the leaky storage model specifies an additional constraint on the time-averaged power consumption. This prevents long-term deviations from the average temperature that may affect the quality of service: alterations to the regular operating cycle can be tolerated only as long as the average temperature is maintained over time. The leaky storage model is an attractive systemlevel representation of TCL flexibility, because it is both exact and sufficient in the limit of a large device population: any power consumption pattern that is compatible with the constraints of the leaky storage model is guaranteed to be realisable by the associated TCL population. It can therefore be used by an aggregator or system operator to dispatch TCL demand. 
Finally, in Sec. IV we show how the leaky storage representation can be used to optimally and simultaneously schedule the power consumption and ancillary service provision of clusters of heterogeneous devices. This is done in the context of a case study using eight cold appliance classes, which provide four ancillary services and energy arbitrage. It demonstrates how physical device characteristics influence the relative ability to provide selected services. The results are validated using Monte Carlo simulations of domestic refrigerators being controlled by the allocated power profile. It is also shown how increasing the number of control clusters may increase the profitability by matching control signals more closely to the capabilities of individual devices.

\section{B. Related Work}

In recent years, the importance of modelling and controlling aggregate TCL dynamics has been widely recognised. Most control strategies $[9,10,11,12,13]$ have assumed the availability of a real-time communication infrastructure to schedule individual TCL states. The control of TCLs is usually implemented centrally using discretised ('bin') models, but this population level description is unwieldy for inclusion in optimisation tools. Mathieu et al [13] therefore proposed to model aggregate TCLs as an effective thermal energy storage unit. This representation enables the computation of near-optimal control trajectories for energy arbitrage, but the model itself and its parameters were approximated, thus impeding accurate control.

Hao et al. have recently presented a generalised battery model for aggregate TCLs, in conjunction with a stack-based feedback control strategy that requires real-time communication with all appliances. [3]. The dynamic equation of the generalised battery model is equivalent to the dynamic equation of the leaky storage model proposed in this work, but the associated power constraints are substantially different, as is the underlying control strategy. The power limits of the generalised battery model are derived using a continuous power model. This may lead to some cases in which reference power profiles that are valid from a 'battery' perspective (e.g. switching off all appliances for 30 minutes) cannot be implemented without violating device temperature constraints. Fundamentally, this reflects the fact that the stack-based does not directly control the shape of the temperature distribution.

In contrast, the leaky storage model developed in this paper makes use of a fully decentralised controller that requires only low-latency channels to communicate desired response patterns [8]. Because the temperature distribution is carefully controlled at all times, it is able to guarantee that solutions of the leaky storage model can be implemented (in the limit of large appliance numbers). Reflecting this guarantee, the power limits of the leaky storage model are more restrictive than those of the generalised battery model. Finally, unlike [3] the leaky storage model proposed in this paper implements a constraint that prevents drift of the average TCL temperature over time. 


\section{Aggregate TCL MOdelling AND CONTROL}

\section{A. Temperature and power}

In its most common implementation a thermostatically controlled appliance $a$ periodically switches between two states: passive cooling/heating (no power consumed) and active heating/cooling (power consumption $P_{o n}^{a}$ ). This system is typically modelled by a first order temperature model ([3], [4], [11], [13]), giving rise to the following ODE for the evolution of the appliance temperature $T^{a}(t)$

$$
\frac{d T^{a}(t)}{d t}=-\frac{1}{\tau^{a}}\left[T^{a}(t)-T_{o f f}^{a}+s^{a}(t) \cdot\left(T_{o f f}^{a}-T_{o n}^{a}\right)\right]
$$

here $s^{a}(t)$ is the binary cooling state of the appliance, 1 (on) or $0($ off $), \tau^{a}$ the thermal time constant, $T_{o f f}^{a}$ the ambient temperature and $T_{o n}^{a}$ the asymptotic cooling/heating temperature, which incorporates a physical model of heat exchange. Considering a population of $N$ TCLs with identical parameters (same $\tau, T_{o n}, T_{o f f}$ ), equation (2b) follows from the linearity of (1); this property is expressed in $(2 a)$.

$$
\begin{gathered}
\frac{1}{N} \sum_{a} \frac{d T^{a}(t)}{d t}=-\frac{1}{N \tau} \sum_{a}\left[T^{a}(t)-T_{o f f}+s^{a}(t) \cdot\left(T_{o f f}-T_{o n}\right)\right] \\
\frac{d \bar{T}(t)}{d t}=-\frac{1}{\tau}\left[\bar{T}(t)-T_{o f f}+\pi(t) \cdot\left(T_{o f f}-T_{o n}\right)\right]
\end{gathered}
$$

In $(2 b), \pi(t)=\frac{1}{N} \sum_{a} s^{a}(t) \in[0,1]$ is the fraction of appliances in the on state. By comparison with (1), we see that the average temperature of a population, $\bar{T}(t)=\frac{1}{N} \sum_{a} T^{a}(t)$, effectively behaves as the hypothetical temperature of a single large appliance with a variable cooling rate. Note that the temperature distribution of a population of identical but independent appliances may also be interpreted as a probability distribution for the temperature of a single appliance with unknown initial conditions [4, 8]. The average properties of such a population are therefore representative of expectation values for a single appliance. This implies that $\pi(t)$ is proportional to the expected power consumption $P^{a}(t)$ of a single appliance:

$$
\pi(t)=E\left[P^{a}(t)\right] / P_{o n}^{a}
$$

From this perspective $(2 \mathrm{~b})$ effectively relates the expected power consumption $E\left[P^{a}(t)\right]$ and temperature $E\left[T^{a}(t)\right]$ of an appliance, and any controller that modulates the expected power consumption thus changes the expected temperature. By considering $d \bar{T}(t) / d t=0,(2 \mathrm{~b})$ also determines the relation between the steady state probability of being in the $o n$ state $\pi_{o}$ (the duty cycle) and the steady state expected temperature $T_{0}$ : 


$$
\pi_{o}=\frac{T_{o f f}-T_{0}}{T_{o f f}-T_{o n}}
$$

Further analysis will be streamlined by the following coordinate transformations:

$$
\Pi(t)=\frac{\pi(t)}{\pi_{0}} \quad \sigma(t)=\frac{T_{o f f}-E\left[T^{a}(t)\right]}{T_{o f f}-T_{o}}
$$

where $\Pi(t)$ is the relative power level and $\sigma(t)$ is a dimensionless measure of stored energy (explored in later sections). The relation between power and temperature $(2 \mathrm{~b})$ can thus be expressed by the simple equation

$$
\frac{d \sigma(t)}{d t}=-\frac{1}{\tau}[\sigma(t)-\Pi(t)]
$$

with the steady state solution $\sigma_{0}=\Pi_{0}=1$. Note that the above analysis is applicable to any first order TCL. The particular control strategy used is only relevant to the extent that it determines the value of $T_{0}$.

\section{B. Controller and quality of service constraints}

The primary function of a TCL is to maintain a compartment close to a desired temperature $T_{0}$. Fluctuations around this target must be limited, resulting in bounds on $\sigma(t)$. In addition, both $\sigma(t)$ and $\Pi(t)$ may be further constrained by the TCL controller implementation.

In this work, we assume that the TCL actions are coordinated by a TCL controller that is based on the design proposed in [8]. It is a decentralised controller in which each appliance independently responds to the control signal $\Pi(t)$ and stochastically modulates its power consumption in accordance with (3), so that the aggregate power consumption of $N \gg 1$ identical appliances is $P(t)=N \pi_{0} P_{o n}^{a} \Pi(t)+O\left(N^{-1 / 2}\right)$. The temperature-linear controller in [8] was designed with analytical simplicity in mind, limiting it to control actions that consist of initial reductions in aggregate power consumption, optionally followed by an increase to recover the steady state temperature $E\left[T^{a}\right]=T_{0}$. In its stated form it is limited to the operating regime summarised by $T_{0} \leq$ $E\left[T^{a}\right] \leq T_{\max }$. To increase the flexibility that is made available to the system we pair it with an alternative mirrored control strategy for the regime $T_{\min } \leq E\left[T^{a}\right] \leq T_{0}$. Details of this procedure are given in the Appendix. Below we summarise the properties of the resulting coupled controller.

In the absence of a control signal the controller acts as a regular deadband controller that keeps the appliance temperature within an acceptable interval $\left[T_{\min }, T_{\max }\right]$. In addition, it guarantees that no appliance ever exceeds this admissible temperature interval as a result of a control action. In terms of the dimensionless parameter $\sigma(t)$ defined in (5) this implies

$$
\sigma_{\min } \leq \sigma(t) \leq \sigma_{\max }
$$

In combination with (6), this constraint limits the duration and extent of changes in the collective power level $\Pi(t)$. The accessible 
range of instantaneous power levels is given by (details in the Appendix)

$$
\Pi_{\min } \leq \Pi(t) \leq \Pi_{\max }
$$

\section{The Leaky Storage Model For Heterogeneous Appliances}

In practice, large groups of TCLs are rarely homogeneous. The objective of this section is to derive an aggregate energy storage model with associated constraints on energy and power levels; it should provide a comprehensive high-level representation of the aggregate flexibility of a large population of heterogeneous TCLs operated with the control strategy described in Sec. II-B. We require the model to be sufficient in the sense that any power consumption profile $\Pi(t)$ that is compatible with the model is guaranteed to be realisable (in expectation) by individual TCLs. This will enable a separation of TCL control problems into two stages: first, the storage representation is used to compute an optimal aggregate response; second, the desired solution is transmitted to individual TCLs, which implement it in a decentralised fashion.

\section{A. Aggregate power control of heterogeneous appliances}

We consider the restrictions imposed by heterogeneous appliances. These will take the form of a lowest-common-denominator model for the appliance capabilities without being unnecessarily conservative. In the following, the superscript $a$ is used to indicate appliance-specific parameters.

\section{1) Aggregate power consumption}

Under the considered control strategy, each appliance individually targets the desired relative power curve $\Pi(t)$, so that the total power consumption equals

$$
P(t)=\hat{P}_{0} \Pi(t)
$$

with

$$
\hat{P}_{0}=\sum_{a} \pi_{0}^{a} P_{o n}^{a}
$$

the superscript $a$ is used to indicate appliance-specific parameters.

\section{2) Instantaneous power consumption}

Equation (8) limits the instantaneous values of the relative power signal $\Pi(t)$, so that any signal that is compatible with all appliances must necessarily satisfy:

$$
\widehat{\Pi}_{\min } \leq \Pi(t) \leq \widehat{\Pi}_{\max }
$$

with 


$$
\widehat{\Pi}_{\min }=\max _{a} \Pi_{\min }^{a} \quad \widehat{\Pi}_{\max }=\min _{a} \Pi_{\max }^{a}
$$

\section{3) Long-term power consumption}

At the opposite time scale we consider the ability to sustain prolonged high/low power levels. This is achieved in the steady state regime where $\Pi(t)$ changes slowly, so that $\sigma(t) \approx \Pi(t)$ (see $(6))$. The limits on slow power signals are thus directly coupled to limits on $\sigma$. Our sufficient model must therefore satisfy

$$
\begin{gathered}
\hat{\sigma}_{\min } \leq \sigma(t) \leq \hat{\sigma}_{\max } \\
\hat{\sigma}_{\min }=\max _{a} \sigma_{\min }^{a} \\
\hat{\sigma}_{\max }=\min _{a} \sigma_{\max }^{a}
\end{gathered}
$$

The relations above prevent the relative power profile from assuming those levels that, if sustained indefinitely, would eventually cause some appliances to become too warm or too cold.

\section{4) Medium-term power consumption}

The final parameter is related to a TCL's ability to track a dynamic signal $\Pi(t)$ that satisfies (10) and (11). This flexibility is limited by the appliance's time constant $\tau^{a}$ and energy constraints $\sigma_{\min }^{a}, \sigma_{\max }^{a}$, and it may be characterised by the minimum time it takes to traverse its accessible energy range. We denote the solution of (6) for appliance $a$ and a constant power level $\Pi$ as

$$
\sigma\left(t ; \sigma^{a}(0), \Pi, \tau^{a}\right)=\left(\sigma^{a}(0)-\Pi\right) e^{-t / \tau^{a}}+\Pi \text {. }
$$

The medium-term energy dynamics of each device $a$ are characterised by two worst-case traversal times: from the population's high energy limit to the appliance's low energy limit and from the population's low energy limit to the device's high energy limit. The worst case decreasing-energy scenario occurs when we start from a constant signal $\Pi(t \leq 0)=\hat{\sigma}_{\max }$, so that $\forall a: \sigma^{a}(0)=$ $\hat{\sigma}_{\text {max }}$, followed by a sudden decrease to $\Pi(t>0)=\widehat{\Pi}_{\text {min }}$. Device $a$ can sustain this power level for a time $t_{\text {down }}^{a}$ until the lower energy bound is reached, implicitly defined by $\sigma\left(t_{\text {down }}^{a} ; \hat{\sigma}_{\max }, \widehat{\Pi}_{\min }, \tau^{a}\right)=\sigma_{\min }^{a}$ :

$$
t_{\text {down }}^{a}=\tau^{a} \ln \left[\frac{\hat{\sigma}_{\max }-\widehat{\Pi}_{\min }}{\sigma_{\min }^{a}-\widehat{\Pi}_{\min }}\right]
$$

This quantity expresses the combined flexibility offered by the time constant $\tau^{a}$ and the energy limit $\sigma_{\min }^{a}$. In order to embed the same amount of flexibility in an envelope model for the entire population, consider that the aggregate lower energy limit is fixed at $\hat{\sigma}_{\text {min }}$ by (11), so that we may define an effective time constant $\tau_{\text {down }}^{a *}$ that realises the same crossing time $t_{\text {down }}^{a}$ for the limit $\hat{\sigma}_{\min }:$ 


$$
\tau_{\text {down }}^{a *}=\tau^{a} \frac{\ln \left[\frac{\hat{\sigma}_{\text {max }}-\widehat{\Pi}_{\text {min }}}{\sigma_{\min }^{a}-\widehat{\Pi}_{\min }}\right]}{\ln \left[\frac{\hat{\sigma}_{\max }-\widehat{\Pi}_{\min }}{\hat{\sigma}_{\min }-\widehat{\Pi}_{\min }}\right]}
$$

Note that $\tau_{\text {down }}^{a *} \geq \tau^{a}$ because $\hat{\sigma}_{\min }$ must equal or exceed $\sigma_{\min }^{a}$. Analogously, we may compute an effective energy-increase time constant for appliance $a$ using the equality $\sigma\left(t_{u p}^{a} ; \hat{\sigma}_{\text {min }}, \widehat{\Pi}_{\text {max }}, \tau^{a}\right)=\sigma_{\text {max }}^{a}$. This results in

$$
\tau_{u p}^{a *}=\tau^{a} \frac{\ln \left[\frac{\widehat{\Pi}_{\max }-\hat{\sigma}_{\min }}{\widehat{\Pi}_{\max }-\sigma_{\max }^{a}}\right]}{\ln \left[\frac{\widehat{\Pi}_{\max }-\hat{\sigma}_{\min }}{\widehat{\Pi}_{\max }-\hat{\sigma}_{\max }}\right]} \geq \tau^{a}
$$

An effective time constant for the population model can then be selected by choosing the most restrictive effective time constant across all appliances as follows:

$$
\hat{\tau}=\min _{a}\left(\min \left(\tau_{u p}^{a *}, \tau_{d o w n}^{a *}\right)\right)
$$

It is worth pointing out that selecting $\hat{\tau}=\min _{a} \tau^{a}$ would also have been a valid choice (instead of (16)), but it would have been overly conservative because it would not allow appliances with small thermal constants to compensate by having flexible energy bounds [8]. The aggregate flexibility of the heterogeneous TCLs is summarised by six parameters: $\left\{\widehat{P}_{0}, \widehat{\Pi}_{\min }, \widehat{\Pi}_{\max }, \hat{\sigma}_{\min }, \hat{\sigma}_{\max }, \hat{\tau}\right\}$ in combination with the constraints (9)-(11), (16) and the power-energy coupling

$$
\frac{d \sigma(t)}{d t}=-\frac{1}{\hat{\tau}}(\sigma(t)-\Pi(t))
$$

We note that the resulting envelope model establishes a lowest-common denominator for the flexibility of all appliances, but this model does not necessarily represent any specific device. Finally, it is evident that the heterogeneous flexibility representation in (9-11) and (16-17) reverts to that in (6)-(8) when all appliances are identical.

\section{B. Population clustering}

The heterogeneous appliances are controlled by a common power signal $\Pi(t)$ that is constrained by the envelope flexibility model. If devices vary significantly, it may be beneficial to partition the population and tailor the desired response to the capabilities of appliance subgroups. In this section, we describe a method to partition a group of $N$ devices into $k$ clusters, each of which is assigned a separate set of envelope parameters $\hat{P}_{0}, \hat{\sigma}_{\text {min }}, \hat{\sigma}_{\text {max }}, \widehat{\Pi}_{\text {min }}, \widehat{\Pi}_{\text {max }}$ and $\hat{\tau}$. The partitioning is performed using the k-means clustering algorithm, where devices are clustered based on their distance in parameter space. The power consumption $P_{0}^{a}$ is not relevant for the ability to track relative power signals $\Pi(t)$, so it may be ignored. Furthermore, as discussed in Sec. IIIA4, the time constant $\tau^{a}$ alone is not sufficient to characterise a TCL's ability to track a power profile in the medium-term. Instead, we define a per-appliance charge/discharge times that define how long the TCLs can operate at their maximum/minimum power 
level, starting from the steady state $\sigma(0)=1$. Using (12), these times are implicitly defined by $\sigma\left(t_{\text {charge }}^{a} ; 1, \Pi_{\max }^{a}, \tau^{a}\right)=\sigma_{\max }^{a}$ and $\sigma\left(t_{\text {discharge }}^{a} ; 1, \Pi_{\min }^{a}, \tau^{a}\right)=\sigma_{\min }^{a}$, resulting in

$$
t_{\text {charge }}^{a}=\tau^{a} \ln \left[\frac{\Pi_{\max }^{a}-1}{\Pi_{\max }^{a}-\sigma_{\max }^{a}}\right] \quad t_{\text {discharge }}^{a}=\tau^{a} \ln \left[\frac{1-\Pi_{\min }^{a}}{\sigma_{\min }^{a}-\Pi_{\min }^{a}}\right]
$$

The minimum of these two values is used as a cluster parameter

$$
t_{\text {clust }}^{a}=\min _{a}\left(t_{\text {charge }}^{a}, t_{\text {discharge }}^{a}\right)
$$

Each TCL is assigned to one of the $k$ clusters according to its vector $\left[\sigma_{\text {min }}^{a}, \sigma_{\text {max }}^{a}, \Pi_{\text {min }}^{a}, \Pi_{\text {max }}^{a} t_{c l u s t}^{a}\right]$. To avoid biasing clusters in favour of high-valued parameters the observed values for each parameter are scaled to the $[0,1]$ range prior to clustering using the squared Euclidean distance. The envelope parameters (9-11) and (16) are computed for each cluster individually. We emphasize that clustering is used to partition the TCLs into sets of similar appliances; it is not trying to identify strictly separated clusters in parameter space. Hence, there is also no clear optimal cluster count: a reasonable number of clusters must be determined from their impact on subsequent optimisation steps; this will be explored in Section V-B. Finally, we point out that the k-means algorithm is a stochastic algorithm, so that cluster assignments are likely to differ between repeated cluster attempts.

\section{The leaky storage model}

In the power system context, it is instructive to reinterpret the envelope model in (9-11) and (16-17) as an effective energy storage unit. Defining the energy level $S(t)$ as

$$
S(t) \equiv \hat{P}_{0} \hat{\tau} \sigma(t)
$$

and using (9a), (17) may be written as

$$
\frac{d S(t)}{d t}=-\frac{1}{\hat{\tau}} S(t)+P(t)
$$

This offers an interpretation of the aggregate TCLs as a leaky storage unit. This storage unit loses its stored energy with an intrinsic rate $\hat{\tau}^{-1}$ and it is replenished by the variable power consumption $P(t)$. The operational limits in (10) and (11) can be transformed to explicit power and energy constraints of the form $P_{\min } \leq P(t) \leq P_{\max }$ and $S_{\min } \leq S(t) \leq S_{\max }$ using (9) and (20). The steady state energy level is given by $S_{0}=\hat{P}_{0} \hat{\tau}$, and the storage capacity may be approximated as $\Delta S=S_{\max }-S_{\min }$. We note that the leaky storage unit cannot physically discharge electricity; it is only able to decrease or increase its consumption relative to its steady state consumption $\hat{P}_{0}$. An intuitive image of this leaky storage model is depicted in Fig.1. 


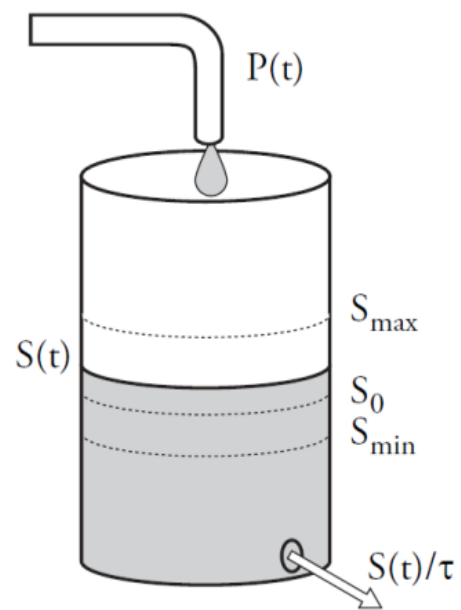

Fig. 1. A physical interpretation of the leaky storage model. The height of a water column is determined by the influx of water from a faucet $(\mathrm{P}(\mathrm{t}) \geq 0)$ and leakage through a hole at the bottom. The flow through the faucet must be controlled in such a way that the water level in the column remains between specified minimum and maximum levels.

In practice, it is likely that different energy bounds will be used for regular (i.e. energy arbitrage) and emergency (i.e. frequency response) services, reflecting the need to maintain stricter temperature limits in everyday use. The full permissible temperature range would then only be used for rare severe frequency events. Differentiated energy bounds are applied as follows:

$$
S_{\min }<S_{\min }^{r} \leq S(t) \leq S_{\max }^{r}<S_{\max }
$$

Finally, it bears mentioning that the provision of demand response services should not excessively impact the primary cooling/heating function of TCLs. Temperature bounds alone are insufficient for this purpose, as appliances could opt to spend all time at the lower or upper temperature bounds. Therefore, we require the expected temperature fluctuations of each appliance to average to zero across a time window of interest $w$. Because population-average temperature and storage level are linearly related by (20) and (5) this implies

$$
\frac{1}{w} \int_{w} S(t) \mathrm{dt}=S_{0}
$$

By combining this with (21) it follows that the average power consumption must also equal the steady state value $\hat{P}_{0}$.

\section{Optimal Multi-SERVICE AllocAtion}

The high-level model of TCLs as a leaky storage unit enables a straightforward embedding in optimisation models. In this section we present its application within a linear optimisation framework for the simultaneous allocation of multiple demand response services. In the following, we consider two distinct types of services. The first of these is energy arbitrage, which enables the TCLs to benefit from energy price fluctuations between different hours of the day. The second type are frequency services that support the balancing of the power system. For the latter we consider only frequency support for infrequent severe events, as opposed to real-time balancing services. Four different frequency services are modelled in this paper, according to the National 
Grid (NG) practice: primary response, high frequency response, secondary and flexible response [14]. The primary and highfrequency response services are short-term services, requiring power consumption to be decreased or increased, respectively, for up to 30 seconds. The secondary response service is a medium term service, requiring a constant power reduction up to 30 minutes. The allocated response for the previous services is constant for the entire period under consideration (in our case 24h). The flexible response is equivalent to secondary response but may be called upon only within two predefined temporal windows $w_{1}$ and $w_{2}$ (typically morning and evening).

The ability of the TCL controller to track an arbitrary reference profile $P(t)$ makes it possible to deliver both energy arbitrage and frequency services simultaneously, so long as their simultaneous provision does not exceed the physical and quality of service constraints of the appliances. In the optimal service allocation problem below the two services are treated in distinct ways. Committing TCLs to perform energy arbitrage implies actual changes in the power consumption profile. This is in contrast to the provision of frequency services, for which the TCLs receive an availability fee even when the service is not called upon. In the following we will assume that activation of frequency services is rare, so that the costs and fees associated with their utilisation are negligible. As a result, the only important consideration for frequency services is that the TCLs maintain sufficient power and energy margins at all times to ensure the deliverability of the frequency services they have committed to without breaching power and energy constraints.

\section{A. The Optimisation Problem}

We consider the optimal allocation of multiple services on a generic day, represented by a 24 -hour period ( $\mathrm{w}=24$ hours) with periodic boundary conditions. It is divided into $m=48$ periods $i$ of $\Delta \mathrm{t}=30$ minutes. Solving (21) on the interval $[0, t]$ and imposing constant electrical power consumption $P_{i}$ within each interval $i$ results in the discrete energy evolution equation:

$$
S_{i+1}=S_{i} \cdot e^{-\frac{\Delta t}{\hat{\tau}}}+\hat{\tau} P_{i} \cdot\left(1-e^{-\frac{\Delta t}{\hat{\tau}}}\right)
$$

where $S_{i}$ and $S_{i+1}$ are the energy levels [MWh] at the beginning and end of interval $i$, respectively. We choose to formulate the problem so that the set of discrete energy levels $\mathcal{S}=\left\{S_{i}\right\}_{i=1}^{m}$ are decision variables. The corresponding discrete power levels $P_{i}$ can be computed by inverting (24), made explicit by the notation $P_{i}(\mathcal{S})$. Note that at each step $i$ the quantities multiplying $S_{i}$ and $P_{i}$ in (24) are constants. TCLs are modelled collectively as a price-taking leaky storage unit, so the optimisation takes the form of payment minimization problem that considers the cost of energy consumption minus any availability fees for frequency services. It is assumed that prices are known at the time the optimisation is performed. The decision variables are the energy levels $\mathcal{S}$ [MWh] and the committed frequency services $P^{p}$ (primary response), $P^{h}$ (high frequency response), $P^{s}$ (secondary response) and $P^{f 1} \&$ $P^{f 2}$ (flexible response) [MW]. 


$$
\min _{\delta, \mathrm{P}^{\mathrm{p}}, \mathrm{P}^{\mathrm{s}}, \mathrm{P}^{\mathrm{h}}, \mathrm{P}^{\mathrm{f}}}\left\{\sum_{i=1}^{\mathrm{m}} \rho_{\mathrm{i}} \cdot \mathrm{P}_{\mathrm{i}}(\mathcal{S}) \cdot \Delta \mathrm{t}-\mathrm{h}^{\mathrm{f}} \cdot\left(\mathrm{w}_{1} \cdot \mathrm{P}^{\mathrm{f} 1}+\mathrm{w}_{2} \cdot \mathrm{P}^{\mathrm{f} 2}\right)-\mathrm{w}\left(\mathrm{h}^{\mathrm{p}} \cdot \mathrm{P}^{\mathrm{p}}-\mathrm{h}^{\mathrm{h}} \cdot \mathrm{P}^{\mathrm{hf}}-\mathrm{h}^{\mathrm{s}} \cdot \mathrm{P}^{\mathrm{s}}\right)\right\}
$$

Subject to:

$$
\begin{array}{lc}
P_{\min } \leq P_{i}(\mathcal{S}) \leq P_{\max } & \forall i \\
S_{\min }^{r} \leq S_{i} \leq S_{\max }^{r} & \forall i \\
\frac{1}{m} \sum_{j=1}^{m} S_{j}=S_{0} & \\
0 \leq P^{p} \leq P_{i}(\mathcal{S})-P_{\text {min }} & \\
0 \leq P^{h} \leq P_{\max }-P_{i}(\mathcal{S}) & \forall i \\
P^{s} \geq 0 & \forall i \\
P^{f 1} \geq 0 & \\
P^{f 2} \geq 0 & \\
P^{s}+P^{f 1} \cdot \mathbb{I}_{i \epsilon w_{1}}+P^{f 2} \cdot \mathbb{I}_{i \epsilon w_{2}} \leq P_{i}(\mathcal{S})-P_{\min } & \\
P^{s}+P^{f 1} \cdot \mathbb{I}_{i \epsilon w_{1}}+P^{f 2} \cdot \mathbb{I}_{i \epsilon w_{2}} \leq P_{i+1}(\mathcal{S})-P_{\min } & \forall i \\
S_{i+1}-\hat{\tau}\left(1-e^{-\frac{\Delta t}{\hat{\tau}}}\right) \cdot\left(P^{s}+P^{f 1} \cdot \mathbb{I}_{i \epsilon w_{1}}+P^{f 2} \cdot \mathbb{I}_{i \epsilon w_{2}}\right) \geq S_{\min } & \forall i \\
S_{i+2}-\hat{\tau}\left(1-e^{-\frac{\Delta t}{\hat{\tau}}}\right) \cdot\left(P^{s}+P^{f 1} \cdot \mathbb{I}_{i \epsilon w_{1}}+P^{f 2} \cdot \mathbb{I}_{i \epsilon w_{2}}\right) \geq S_{\min } & \forall i
\end{array}
$$

For a given price profile, (25-32) fully define a linear optimisation problem that determines the optimal allocation of services to maximise the aggregate TCL profit across the 24-hour window. The electricity price $\rho_{i}$ per period $i$ and the availability fees $h^{x}$ for each frequency service $x$ are expressed in $£$ /MWh. The solution is bounded by the controller's power constraints (26) and the energy constraints for regular operation (27). In addition, (28) enforces the average temperature to equal the steady state temperature. This constraint follows from inserting (21) in (23) and using (24) to eliminate $P_{i}$ in each interval. Adequate reserves for primary response and high frequency response allocations are enforced by (29). The same aim characterises (30-31), with regard to secondary and flexible response inside and outside $w_{1}\left(P^{f 1}\right)$ and $w_{2}\left(P^{f 2}\right)$. The indicator function $\mathbb{I}_{s}$ returns 1 if statement $s$ is true, and 0 otherwise. It is assumed that the provision of primary and high frequency response for 30 seconds has a negligible impact on energy levels. A frequency event may occur at any time within an interval, and the secondary/flexible response commitments must be provided for 30 minutes from that moment onward. For this reason, we must ensure that the services agreed for interval $i$ can also be provided for the interval $i+1$, resulting in constraint $\left(31_{\mathrm{b}}\right)$. Equations (32) ensure that calling upon the 
secondary and flexible frequency services will not conflict with the emergency lower energy limit $S_{m i n}^{e}$. Similar to the power constraint, the energy constraint is also enforced for the subsequent interval $i+1$.

\section{B. Case Study}

We consider eight distinct types of refrigeration units across the domestic and commercial sectors. Refrigeration units represent a substantial base load and are connected to the grid at nearly all times, making them suitable for providing frequency services around the clock. For the domestic sector we distinguish refrigerators, freezers and fridge-freezers, while within the commercial domain we take into account bottle coolers (with glass doors), upright refrigerators, upright freezers, and two different multideck refrigerators (open retail units). Table I summarises their characteristics; each appliance class is based on a representative appliance (first seven columns), the parameters of which are independently varied by $+/-15 \%$ to generate a heterogeneous set of appliances for that class. Aggregate envelope parameters for each class (last six columns) are computed using (9-11) and (16).

TABLE I

CHARACTERISTICS OF SELECT TCLS

\begin{tabular}{|c|c|c|c|c|c|c|c|c|c|c|c|c|c|}
\hline TCL class & \multicolumn{6}{|c|}{ Representative appliance parameters } & & \multicolumn{6}{|c|}{ Heterogeneous cluster parameters } \\
\hline Domestic freezer & 5.1 & -15 & -21 & -135 & 20 & 100 & -17.9 & 4.4 & 24.7 & 0.54 & 2.03 & 0.94 & 1.06 \\
\hline Bottle cooler & 5.5 & 10 & 4 & -1 & 25 & 200 & 6.8 & 4.7 & 139.6 & 0.66 & 1.08 & 0.88 & 1.10 \\
\hline Upright fridge & 15.2 & 4 & 1 & -28 & 23 & 150 & 2.5 & 12.9 & 60.0 & 0.54 & 1.51 & 0.95 & 1.05 \\
\hline Upright freezer & 19.2 & -18 & -22 & -84 & 23 & 250 & -20.0 & 16.3 & 100.4 & 0.53 & 1.48 & 0.97 & 1.03 \\
\hline Multideck 1 & 0.6 & 6.5 & 1.2 & -5 & 25 & 750 & 3.7 & 0.5 & 524.0 & 0.62 & 1.11 & 0.91 & 1.08 \\
\hline
\end{tabular}

Default values for domestic unit temperature thresholds and room temperature are taken from [15]. The duty cycle and steady state cycle duration were computed numerically using a second order model, and their values were used to fit $\boldsymbol{T}_{\boldsymbol{o n}}$ and $\boldsymbol{\tau}$ to a first order model within the nominal temperature range. First order dynamic models of the commercial TCLs are derived from [16], considering typical duty cycles and daily consumptions in [17]. The duty cycle $\boldsymbol{\pi}_{\mathbf{0}}$ can be computed from the model parameters using (4). Note that instead of $\boldsymbol{T}_{\max }$ and $\boldsymbol{T}_{\min }$ we vary $\left[\left(\boldsymbol{T}_{\max }-\boldsymbol{T}_{\min }\right) / 2\right]$ and $\left(\boldsymbol{T}_{\max }-\boldsymbol{T}_{\min }\right)$ by $+/-15 \%$ to simulate variability in the set-point temperature and deadband width.

We consider a half-hourly representative price profile that reflects the load and generator dispatch on the Great Britain (GB) system on a winter day (see Fig. 2a). The response services are priced at $h^{p}=6 £ / \mathrm{MWh}, h^{h}=7 £ / \mathrm{MWh}, h^{s}=5 £ / \mathrm{MWh}$ and $h^{f}=8$ $£ / M W h$, in line with NG values [14]. The slightly higher value for $h^{f}$ reflects the desire to incentivize the provision of response services within the two flexible response windows: $w_{1}$ ranges from hour 06:00 to 09:00 and $w_{2}$ from 16:00 to 20:00. 


\section{Results}

\section{1) Service allocation for domestic refrigerators}

Fig. 2b-d shows the optimisation result for a heterogeneous population of domestic refrigerators (here 1MW base load is used for illustration). The results were obtained considering a single cluster of heterogeneous devices (parameters in Table I). The energy profile of the leaky storage unit corresponding to the domestic refrigerators is shown in Fig. 2b. The regularly accessible energy range is assumed to be $75 \%$ with respect to $S_{0}$ of the full accessible range, in accordance with (22). The optimised energy profile (solid black) is high in the morning in response to low prices during the night and mostly low during the afternoon because of high prices during the day. The profile is constrained by the regular energy limits and the average energy constraint (28). Fig.2c illustrates the corresponding optimal power consumption profile (solid black) and compares it to the nominal power consumption (blue).

Fig. $2 d$ illustrates the allocation of frequency services. Supplying short-term services, primary (green) and high frequency response (grey) restricts the power levels accessible for energy arbitrage, but is nevertheless desirable due to the high holding prices for these services. In contrast, the allocation of 30-minute secondary (magenta) and flexible (red) response services impacts both power and energy constraints, and as a result the committed amounts are lower. Lastly, flexible response availability is selected only for $w_{1}$ facilitated by low energy prices in this period. During $w_{2}$ the price reaches its highest value, so it is desirable to deplete the energy reserves and reduce the aggregate power consumption to a minimum. This conflicts with the provision of flexible response in this window.

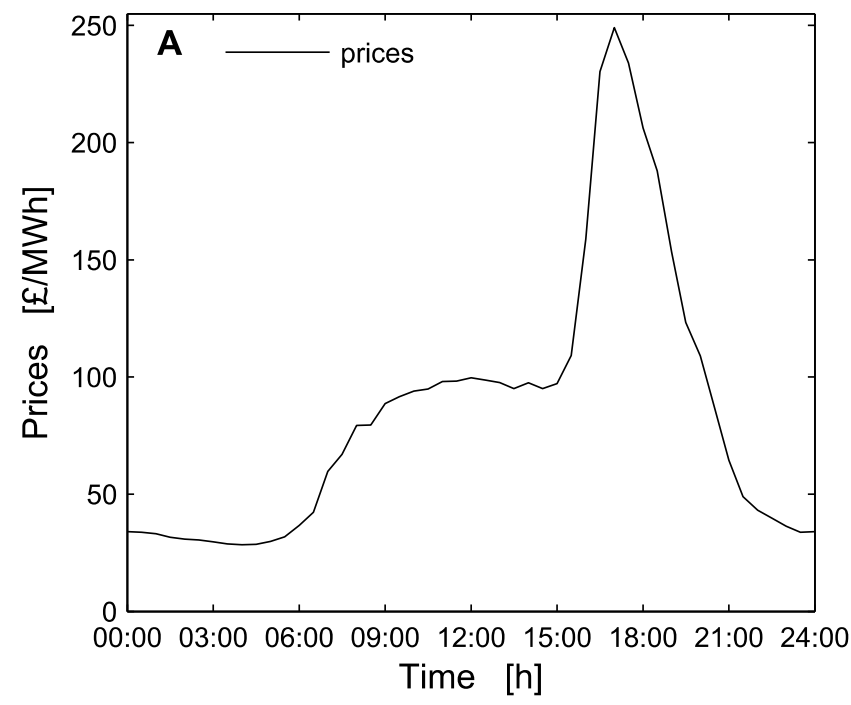



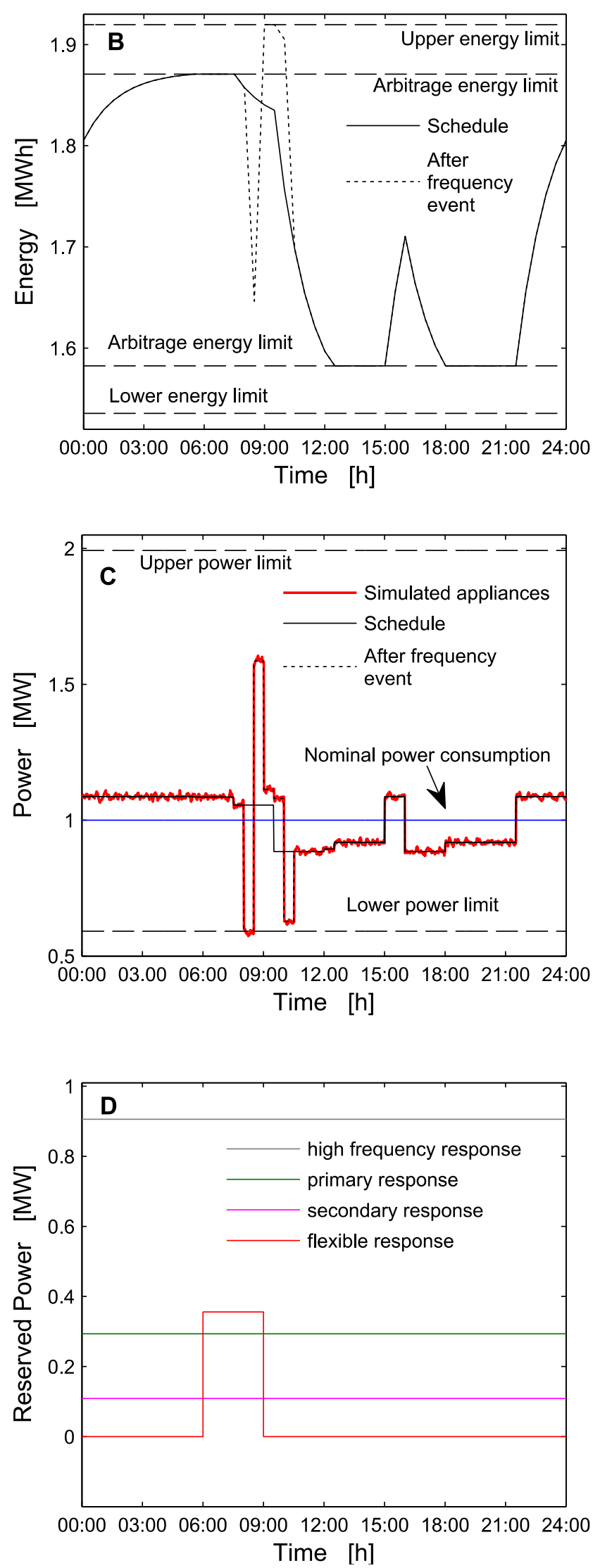

Fig. 2. Optimal service allocation for $1 \mathrm{MW}$ of heterogeneous domestic refrigerators (single-cluster population). (A) Price profile for a representative winter day. (B) Optimal energy profile (solid) and perturbed energy profile (dotted) in case of provision of frequency services. (C) Optimal power profile (solid black) compared to the nominal consumption (blue). The dotted curve is the realised power consumption after provision of frequency services; Monte Carlo simulation 
results based on this solution are shown in red. (D) Optimal allocation of the frequency response services. Problem (30)-(37) was solved using the linear programming routine - linprog - of Matlab on an Intel Core i7-4600U CPU. For a single TCL class the optimisation took 0.34s (using a single cluster), while the same algorithm (including clustering) took $2.07 \mathrm{~s}, 5.84 \mathrm{~s}, 20.40 \mathrm{~s}$ and $225.3 \mathrm{~s}$ when the TCL population was divided into $5,25,100$ and 500 clusters, respectively.

Furthermore, the black dotted curve in Fig. 2b-c illustrates the deliverability of contracted ancillary services and the subsequent recovery of normal operations after their provision. In this example a sudden loss of generation at 08:00 activates the primary, secondary and flexible response services. As a result, the energy level drops and deviates from the scheduled profile. An optimal recovery pattern is computed to force the TCLs to return to the scheduled energy profile as fast as possible, subject to the daily average energy constraint (28). The return trajectory must respect the power constraint (26) and the energy constraints as in (27), but has access to the full energy range instead of the restricted range; this relaxation facilitates the rapid return to the predetermined energy profile. Note that (28) forces the TCLs to behave quite unlike a regular storage unit, because it enforces a period of 'overcharging' after the initial recovery. Finally, it is worth pointing out that different recovery policies can be implemented depending on TSO requirements and commercial arrangements.

\section{2) Validation using device level simulations}

The aggregate flexibility of a cluster of heterogeneous TCLs was summarised as a leaky storage unit; this representation was then used to determine an allocation of services and power consumption that makes optimal use of the available flexibility. In order to validate these results we make use of explicit simulations of device dynamics. 59,524 domestic refrigerator models (1MW steady state load) were initialised with random parameters and random initial temperatures. They were instructed to track the power profile in Fig. 2c, including the frequency response activation (black dotted line). The appliances are controlled in a fully decentralised manner according to the control strategy and algorithm in [8], with enhancements described in the Appendix to this paper. The algorithm was implemented in Mathematica 10. The empirical power consumption of the simulated appliances is shown in Fig. 2c (red line), confirming the accurate tracking of the desired power profile and the feasibility of the solution. Furthermore, Fig. 3 shows the temperature traces of 100 simulated domestic refrigerators during the provision of this response. Temperatures are shown on a scale between $T_{\min }$ and $T_{\max }$, to account for the heterogeneity of the TCL population. It is clear that the controller is indeed non-disruptive, i.e. no device is forced to operate outside its permitted temperature range. 


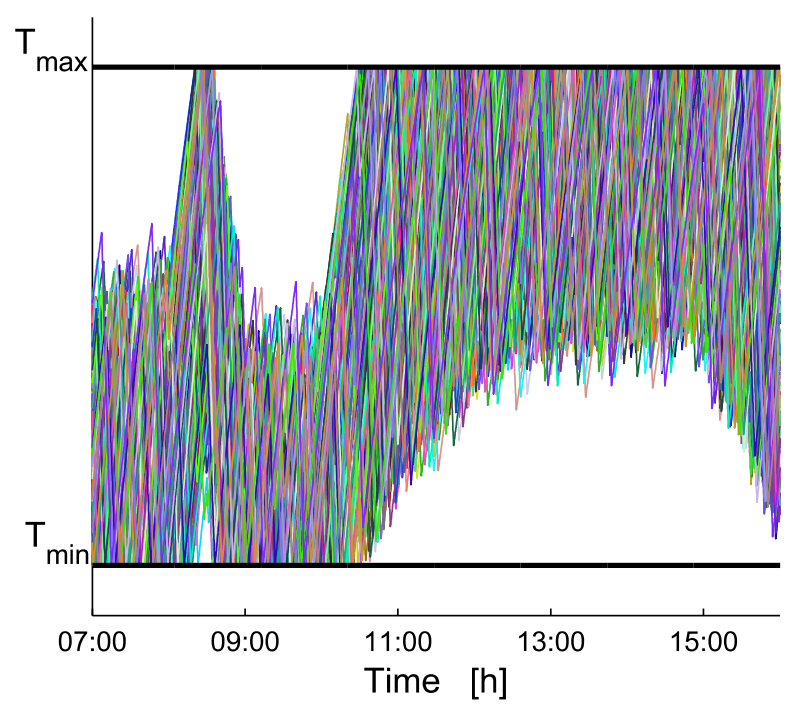

Fig. 3. Temperature profiles of 100 domestic refrigerators with different randomised parameters (solid lines). The black lines are the corresponding thermostat temperature thresholds, which vary per appliance.

\section{3) Profit per device class}

Fig. 4 shows the profits that result from the optimal service allocation of each TCL class. The differences in total profit and its allocation to energy arbitrage and frequency services (different colours) reflect the underlying device capabilities. Domestic appliances make most of their profit from the provision of high frequency response (pink), courtesy of their large power headroom $\left(\Pi_{u p}=\widehat{\Pi}_{\max }-\Pi_{0}\right)$. In contrast, this component is (nearly) absent for bottle coolers and multidecks. Moreover, upright fridges and freezers and bottle coolers can profit significantly from energy arbitrage due to their large effective storage capacities $\Delta S=$ $\hat{P}_{0} \hat{\tau}_{0}\left(\hat{\sigma}_{\text {max }}-\hat{\sigma}_{\text {min }}\right)$, whereas the multidecks perform less well due to their weak thermal insulation (i.e. small $\tau$ ). In general, flexible power limits are advantageous for power-intensive services, whereas energy-intensive services and energy arbitrage benefit from weak temperature constraints or large thermal time constants. The optimal service portfolio ultimately depends on the lucrativeness of individual services and the presence of conflicts and synergies from their interactions. 


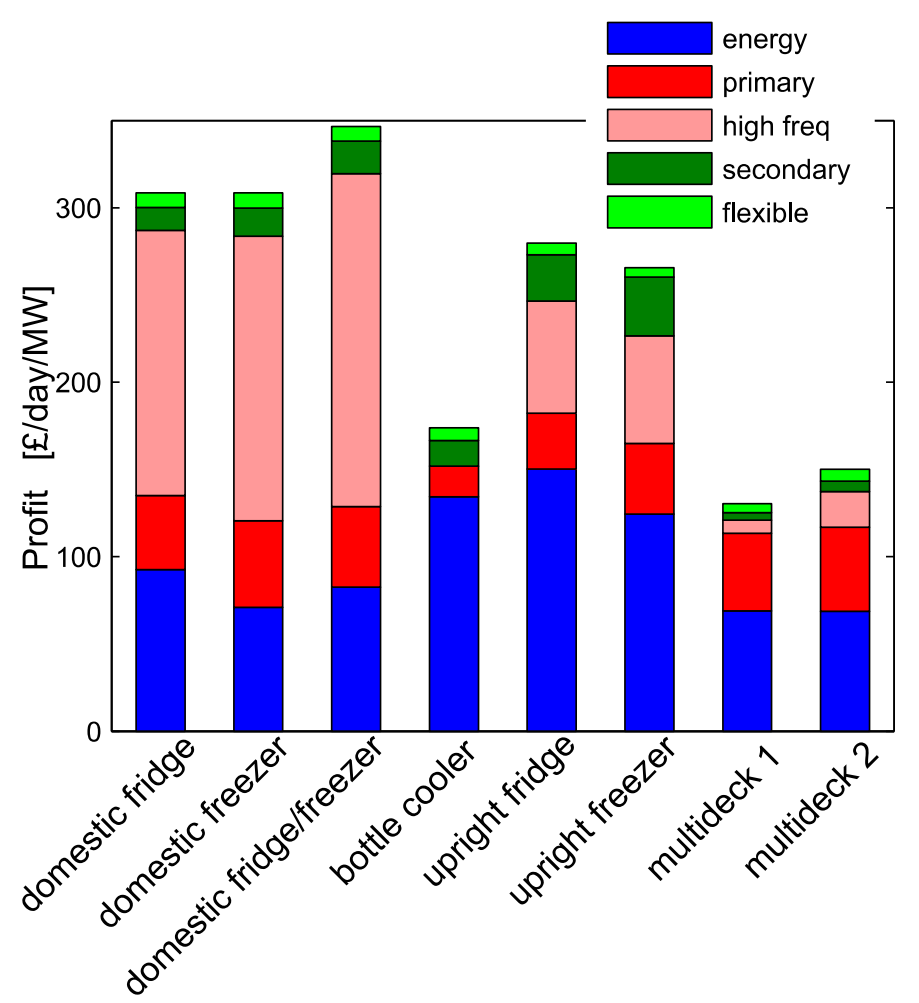

Fig. 4. Daily profit for each TCL class (1MW load; single cluster) realised by energy arbitrage and by selling simultaneous availability for multiple frequency services. The profit derived from the provision of a frequency service is the product of the power reserved for that service (Fig. 2d), the corresponding availability fee and the applicable service window. The profit due to energy arbitrage is calculated as the additional expense incurred by TCLs at their nominal power level.

The additional profit that can be obtained by dividing $N=1 M W / \widehat{P}_{0}$ devices of each class into $k$ of clusters is illustrated in Fig. 5. The service allocation problem (25-32) was solved for each cluster individually and its contribution to the profit assessment of the whole population was weighted by cluster size, i.e. the number of devices belonging to that cluster. The solid lines show that the average profit increases significantly with the number of clusters: using 25 clusters per class of devices boosts the savings by approximately $20 \%$; an increase to 500 clusters would further increase the profits to approximately $30 \%$ (compared to a nonclustered approach). 


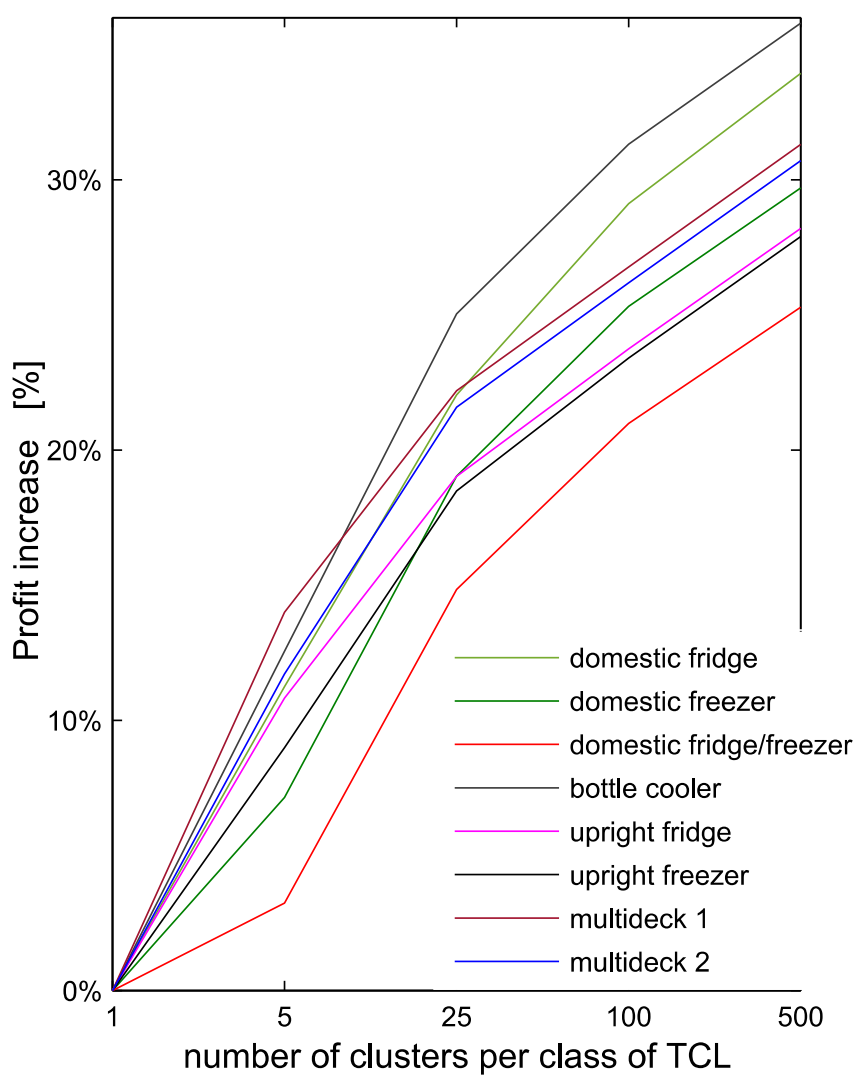

Fig. 5. Profit increase realised by splitting the device population of each class into clusters according to their abilities. Results were averaged over 100 repetitions of the clustering procedure with random sets of initial appliance parameters.

\section{SUMMARY AND DISCUSSION}

In this paper we have demonstrated how a large heterogeneous collection of thermostatically controlled loads can be modelled as a single leaky energy storage unit. The proposed storage model is a high-level representation of aggregate TCL flexibility that is particularly insightful for embedding in optimization tools. The accessible storage levels $S(t)$ and power consumption levels $P(t)$ of this unit are limited by the appliances' thermal models, their temperature constraints and the control strategy adopted. The model has been introduced in the context of cold appliances, but it applies equally to heating appliances. The leaky storage model is exact for large TCL populations with linear thermal models that are controlled by the controller in [8], and it guarantees the deliverability of a compatible power consumption pattern by a heterogeneous collection of appliances.

The leaky storage representation allows for an embedding in revenue-maximising scheduling models. In this paper, we have demonstrated an application to simultaneous allocation of energy consumption and ancillary services. The results obtained for eight classes of cold appliances show that differences in physical models and quality of service constraints lead to very diverse profit potential. In all cases, profits can be enhanced significantly by clustering appliances with similar capabilities.

The leaky storage model (based on envelope equations (9-11) and (16)) may also be used to increase the robustness of TCL 
dispatch solutions to unknown variations in model parameters. Even when the true appliance parameters are not accurately known when services are allocated, the appliances will still be able to provide the desired response provided that 1) each appliance has learned its true model by the time the response is executed, and 2) the envelope encompasses the realised models of all appliances. This will be the case even when the models change over time (e.g. day-night cycle), as long as the change in model parameters is sufficiently slow compared to the appliances' intrinsic dynamics.

The results presented in this paper suggest a number of pathways for further development. First, the underlying control strategy can be further enhanced to provide access to a larger range of instantaneous power levels. Similarly, whereas the control strategy is currently formulated for first order thermal models, initial investigations suggest that it could be extended to higher order models for increased accuracy. Such models could provide more control over the temperature dynamics within TCLs, and could be used to impose more specific temperature constraints, e.g. on food temperature. Moreover, the optimal service allocation case study may be enhanced in a number of ways. It currently assumes that responsive TCLs are not sufficiently numerous to affect energy and ancillary service prices. However, the future grid is expected to feature significant levels of TCL demand response, raising the possibility to exploit the collective flexibility offered by TCLs to relax constraints on the generators' dispatch. An initial exploration of this aspect is the subject of [18]. The 24-hour periodic optimisation of service allocation could also be extended to a continuous optimal control problem on longer time scales, potentially taking into account the fact that the provision of ancillary services usually requires longer term contracts. Finally, the assumption that income and losses due to the utilisation of these services can be neglected may need to be reconsidered for future power systems as the frequency of utilisation increases.

\section{ACKNOWLEDGMENTS}

The authors thank A. De Paola for enlightening discussions, and the anonymous reviewers for helpful suggestions. ST has received funding from the European Union Seventh Framework Programme (FP7/2007-2013) under grant agreement n 274387.

\section{APPENDIX: CONTROLLER DETAILS}

\section{A. Power limits}

The temperature-linear controller in [8] for the temperature range $E\left[T^{a}\right] \in\left[T_{0}, T_{\max }\right]$ is defined by the net heating rate profile

$$
v^{\text {regular }}(T, t)=\alpha \beta^{\text {regular }}(t)\left(T-T_{\max }\right)
$$

which results in a contraction or expansion of the temperature distribution around $T_{\max }$. Note that we the same notation from [8], where $\alpha=1 / \tau$. The expressions of constant power limits in [8] are 


$$
\begin{gathered}
\Pi_{\min }^{\text {regular }}=\frac{\left(T_{\text {off }}-T_{\max }\right)\left(T_{0}-T_{\text {min }}\right)}{\left(T_{\max }-T_{\min }\right)\left(T_{o f f}-T_{0}\right)} \\
\Pi_{\max }^{\text {regular }}=\frac{\left(T_{\max }-T_{0}\right)\left(T_{\max }-T_{o n}\right)+\left(T_{o f f}-T_{\max }\right)\left(T_{\max }-T_{\min }\right)}{\left(T_{\max }-T_{\min }\right)\left(T_{o f f}-T_{0}\right)} .
\end{gathered}
$$

A complementary controller for temperatures $E\left[T^{a}\right] \in\left[T_{\min }, T_{0}\right]$ is constructed by choosing

$$
v^{\text {mirror }}(T, t)=\alpha \beta^{\text {mirror }}(t)\left(T-T_{\text {min }}\right)
$$

which implements a mirrored compression around the lower temperature bound $T_{\text {min }}$. The power limits can be computed using the methodology in [8], resulting in power limits where $T_{\min }$ and $T_{\max }$ have been interchanged.

$$
\begin{gathered}
\Pi_{\text {min }}^{\text {mirror }}=\frac{\left(T_{o f f}-T_{\min }\right)\left(T_{0}-T_{\text {max }}\right)}{\left(T_{\min }-T_{\max }\right)\left(T_{o f f}-T_{0}\right)} \\
\Pi_{\text {max }}^{\text {mirror }}=\frac{\left(T_{\min }-T_{0}\right)\left(T_{\min }-T_{o n}\right)+\left(T_{o f f}-T_{\min }\right)\left(T_{\min }-T_{\max }\right)}{\left(T_{\min }-T_{\max }\right)\left(T_{o f f}-T_{0}\right)}
\end{gathered}
$$

Together, the controller implementations span the operating temperature range $E\left[T^{a}\right] \in\left[T_{\min }, T_{\max }\right]$, and appliances can switch between both strategies when $E\left[T^{a}\right]$ crosses $T_{0}$. Operational power limits that are achievable across the entire operating range are defined as

$$
\begin{aligned}
& \Pi_{\min }=\max \left(\Pi_{\min }^{\text {regular }}, \Pi_{\min }^{\text {mirror }}\right) \\
& \Pi_{\max }=\min \left(\Pi_{\max }^{\text {regular }}, \Pi_{\max }^{\operatorname{mirror}}\right)
\end{aligned}
$$

\section{B. Discontinuous transitions}

The simulation algorithm in [8] computes the required device-level switching actions for a continuously changing power profile, but discontinuities require special consideration. Sudden changes in power consumption imply step changes in the velocity profile (A1) or (A3) and result in the requirement to immediately switch on/off a fraction of the refrigerator population. A similar situation occurs when switching between the 'regular' and 'mirrored' operating regimes of the controller. In this case - even if the desired power consumption remains the same - appliances across the temperature range must switch on or off to realise the immediate change in heating profile from (A1) to (A3) or vice versa. The required switching probabilities are computed below.

We define a time-dependent probability density $f(T, t)=f_{\text {on }}(T, t)+f_{\text {off }}(T, t)$ for the fridge temperature, where $f_{\text {on }}$ and $f_{\text {off }}$ represent the subpopulations of fridges that are in the on and off state, respectively. The probability of a random appliance with temperature $T$ at time $t$ to be in the on state is therefore $\operatorname{Pr}(o n \mid T, t)=f_{\text {on }}(T, t) / f(T, t)$. The PDF $f(T, t)$ can change only slowly, but $f_{\text {on }}(T, t)$ may change abruptly through on-off switching. When the heating profile changes suddenly from $v^{\text {pre }}(T, t)$ to 
$v^{\text {post }}(T, t)$, it follows that the probability to switch off is given by

$$
\operatorname{Pr}(\text { on } \rightarrow \text { off } \mid \text { on }, T, t)=\frac{\max (0, \operatorname{Pr}(\text { on, } \operatorname{pre} \mid T, t)-\operatorname{Pr}(\text { on, post } \mid T, t))}{\operatorname{Pr}(\text { on, } \operatorname{pre} \mid T, t)}=\max \left(1-\frac{f_{\text {on }}^{\text {post }}(T, t)}{f_{\text {on }}^{\text {pre }}(T, t)}, 0\right)
$$

Using equation (14) in [8], this is expressed in terms of $v(T, t)$ as follows:

$$
\operatorname{Pr}(\text { on } \rightarrow \text { off } \mid \text { on }, T, t)=\max \left(1-\frac{v^{\text {post }}(T, t)+\alpha \cdot\left(T-T_{\text {off }}\right)}{v^{p r e}(T, t)+\alpha \cdot\left(T-T_{o f f}\right)}, 0\right)
$$

An equivalent analysis for the probability to switch on results in

$$
\operatorname{Pr}(\text { off } \rightarrow \text { on } \mid \text { off }, T, t)=\max \left(1-\frac{v^{\text {post }}(T, t)+\alpha \cdot\left(T-T_{\text {on }}\right)}{v^{\text {pre }}(T, t)+\alpha \cdot\left(T-T_{\text {on }}\right)}, 0\right)
$$

In the refrigerator controller, these probabilities are evaluated whenever a discontinuity in $v(T, t)$ is encountered, and are used to randomly switch appliances on/off depending on their temperatures.

\section{REFERENCES}

[1] J.Short, D.Infield and L.L. Freris, "Stabilization of Grid Frequency through Dynamic Demand Control," IEEE Transactions on Power Systems, vol. vol.22, no. no.3, Aug 2007.

[2] J. Mathieu, M, Kamgapour, J. Lygeros, G. Andersson and D.Callaway, “Arbitraging intraday wholesale energy market prices with aggregations of thermostatic loads," IEEE Trans. on Power Syst., 2014, in press.

[3] H. Hao, M. Sanandaji, K.Polla and T. Vincent, "Aggregate flexibility of Themostatically controlled loads," IEEE Transactions on Power Systems, vol. 30, no. 1, pp. 189-198, 2015.

[4] D.Angeli and P.S. Kountouriotis, "A stochastich approrach to Dynamic-Demand refrigerator Control," IEEE Trans. Control Syst. Technol., vol. 20, no. 3, pp. 581-592, May 2012.

[5] U.S. Department of Energy, “Load participation in ancillary services,” Dec. 2011.

[6] R.Moreno, R.Moreira and G. Strbac, "A MILP model for optimising multi-service portfolios of distributed energy storage," Applied Energy - Elsevier, 2014.

[7] D.Callaway and S. Hiskens, "Achieving Controllability of Electric Loads," Proceedings of the IEEE, vol. 39, no. 1, pp. 184199, 2011.

[8] S.Tindemans, V.Trovato and G.Strbac, "Decentralised control of thermostatic loads for flexible demand response," IEEE Transactions on Control System Technology, 2015 - in press.

[9] D. Callaway, "Tapping the energy storage potential in electric loads to deliver load followingand regulation, with appliacation to wind energy," Energy Conversion and Management, vol. 50, no. 5, pp. 1389-1400, 2009.

[10] S. Kundu, N, Sinitsyn, S. Backhaus and I.Hiskens, "Modeling and control of thermostaticaly controlled loads," in 17th Power Systems Computation Confernce, Stockholm, 2011. 
[11] C.Perfumo, E. Kofman, J.H. Braslavsky and J. Ward, "Load management:Model-based control for population of thermostatically controlled loads," Energy Conversion and Management, vol. 55, pp. 36-48, Mar. 2012.

[12] W.Zhang, J. Lian, C.Y. Chang and K. Kalsi, "Aggregated Modeling and Control of Air Conditioning Loads for Demand Response," IEEE Transactions on Power Systems, vol. 28, no. 4, 2013.

[13] J.L. Mathieu, S.Koch and D.S.Callaway, "State Estimation and Control of Electric Loads to Manage Real-Time Energy Imbalance," IEEE Trans. Power Syst., vol. 28, no. 1, pp. 430-440, 2013.

[14] National Grid, [Online]. Available: http://www2.nationalgrid.com/uk/services/balancing-services/.

[15] V.Trovato, S.Tindemans and G.Strbac, "Controlling the synchronization and payback associated with the provision of frequency services by dynamic demand," in 22nd CIRED Conference, Sotckholm, 2013.

[16] Williams Refrigeration, [Online]. Available: http://www.williams-refrigeration.co.uk/.

[17] Navigant Consulting, Inc., "Energy Savings Potential and R\&D Opportunities for Commercial Refrigeration,” 2009.

[18] V. Trovato, S. Tindemans and G. Strbac, "Security Constrained Economic Dispatch with Flexible Thermostatically Controlled Loads," in IEEE PES ISGT Europe, Istanbul, 2014. 